\title{
Civilisations
}

Revue internationale d'anthropologie et de sciences

humaines

$54 \mid 2006$

Expériences de recherche en République

démocratique du Congo

\section{La recherche en santé}

Systèmes, acteurs et savoirs en République Démocratique du Congo

Denis Porignon, Philippe Hennart, Michèle Dramaix et Philippe Donnen

\section{(2) OpenEdition}

Journals

Édition électronique

URL : http://journals.openedition.org/civilisations/425

DOI : $10.4000 /$ civilisations.425

ISSN : 2032-0442

Éditeur

Institut de sociologie de l'Université Libre de Bruxelles

Édition imprimée

Date de publication : 1 avril 2006

Pagination : 167-177

ISBN : 2-87263-006-6

ISSN : 0009-8140

Référence électronique

Denis Porignon, Philippe Hennart, Michèle Dramaix et Philippe Donnen, «La recherche en santé », Civilisations [En ligne], 54 | 2006, mis en ligne le 01 avril 2009, consulté le 19 avril 2019. URL : http:// journals.openedition.org/civilisations/425; DOI : 10.4000/civilisations.425 


\title{
La recherche en santé
}

\section{Systèmes, acteurs et savoirs en République Démocratique du Congo}

\section{Denis PORIGNON, Philippe HENNART, Michèle DRAMAIX, Philippe DONNEN}

\begin{abstract}
Résumé : Les activités de recherche font partie du modèle de coopération développé par le Cemubac. Les fondements de la recherche sont établis sur le renforcement du fonctionnement des systèmes et des services de santé aboutissant à l'amélioration de la qualité des prestations offertes aux communautés. Qu'elle soit quantitative ou qualitative, cette recherche à composante participative élevée a pu se développer à la faveur d'un partenariat établi avec les acteurs, qu'ils soient professionnels ou bénéficiaires. Elle s'appuie largement sur une implication de cadres institutionnels formés à cet effet et repose sur une présence inscrite dans la durée. La recherche menée au Kivu a été conduite depuis plus de quinze ans dans un climat de dégradation de l'environnement économique, social et surtout sécuritaire. Appareil d'acquisition de connaissances, la recherche développée au Kivu reste avant tout orientée vers l'amélioration de l'offre des services.
\end{abstract}

Mots-clés : systèmes de santé, malnutrition, formation, Kivu.

Summary: Research activities are part of the cooperation model developed by the Cemubac. The foundation for the research is based on strengthening the capacity of health systems and services resulting in the improvement of care offered to communities. The research (quantitative and qualitative) has a high participatory dimension and was able to develop thanks to partnerships with professionals and beneficiaries. It relies heavily on the implication of national public staff trained specifically for this work and on long-term presence in the area. The work in Kivu has continued over the past fifteen years despite a deteriorating social, economic and security context. As an organism for acquiring knowledge, the Cemubac's work in Kivu is primarily geared towards improving health services.

Key words: health systems, malnutrition, training, Kivu. 


\section{Introduction}

T e Cemubac est une organisation non gouvernementale (ONG) attachée à Ll'Université libre de Bruxelles et spécialisée dans la coopération au développement. Elle comporte un certain nombre de sections dont la section « Soins de santé primaires et nutrition » $(\mathrm{SSP} \& \mathrm{~N})$ qui propose le présent travail. Cette section a développé un modèle de coopération scientifique et médicale dont les caractéristiques sont au nombre de trois :

1. Appui au fonctionnement global des systèmes de santé;

2. Développement du programme de formation du personnel de santé;

3. Promotion d'un programme de surveillance épidémiologique et de recherche appliquée.

Les caractéristiques de ce modèle tiennent compte d'une part, des priorités identifiées par le Cemubac et ses partenaires, d'autre part, des spécificités d'une organisation universitaire de coopération.

Le premier axe d'intervention du Cemubac envisage donc le soutien aux structures sanitaires, qu'elles soient de base, de référence, ancrées au niveau local, provincial ou national. Cela se traduit, entre autres, depuis plus de 30 ans, par un soutien à l'Hôpital pédiatrique de Lwiro couvrant environ 35000 personnes, où chaque année plus de mille enfants sont hospitalisés et traités principalement pour malnutrition grave, et par le support financier, technique et logistique de trois zones de santé (Kirotshe, Masisi et Rutshuru) couvrant environ un million de personnes pour lesquelles le Cemubac organise avec différents partenaires locaux et internationaux des services et des soins de santé de base dans une soixantaine de centres de santé et trois hôpitaux de référence totalisant 500 lits d'hospitalisation.

Le deuxième axe est celui de la formation. Qu'elle soit de base ou continuée, de nombreux professionnels de santé, médecins, administrateurs, infirmiers, nutritionnistes, etc., ont bénéficié en République Démocratique du Congo (RDC), en Afrique ou en Europe, de formations leur permettant d'asseoir leurs capacités en développant leurs compétences techniques dans les domaines de la médecine clinique mais également de la santé publique, gestion, nutrition, développement rural, comptabilité, etc.

Le troisième axe est celui de la recherche appliquée, développée comme un instrument d'acquisition de connaissances mais surtout comme un outil de résolution de problèmes opérationnels de terrain, de motivation du personnel et de contribution à la cohésion des interventions menées sur le terrain.

L'objectif du présent travail est plus de présenter la logique de recherche développée progressivement par le Cemubac au Kivu et de montrer comment s'est opérée l'implication des différents acteurs plutôt que d'insister sur les résultats proprement dits. Ces derniers sont accessibles à travers les différentes publications référencées tout au long du texte.

\section{Méthodes utilisées pour mener les recherches au Kivu}

Les outils développés sont principalement quantitatifs mais font également appel aux méthodes qualitatives (figure 1). Toutes les recherches quantitatives qui ont été menées se sont appuyées sur des protocoles préétablis et écrits avec toute la rigueur scientifique exigée pour la réalisation d'études épidémiologiques valables. Les analyses statistiques 
ont été effectuées en utilisant une large panoplie d'outils allant des statistiques usuelles aux courbes ROC, analyses de clusters et modèles multivariables tels que les régressions multiples et logistiques.

Il importe de souligner qu'une partie des travaux (relatifs aux aspects de lutte contre des maladies spécifiques) a été réalisée sur base d'enquêtes, d'études et de recueil d'information spécifiquement conçus pour les besoins de la recherche. Cette dernière a alors été menée selon un protocole préétabli qui devait être respecté.

\section{Figure 1 : Méthodes utilisées pour conduire les différentes recherches}

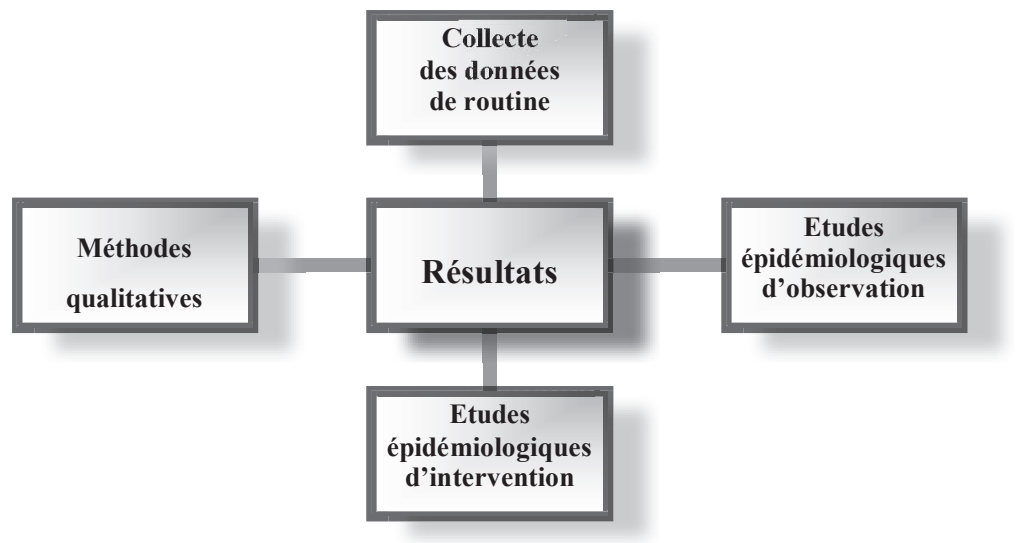

L'autre partie, portant plus sur les aspects d'organisation des services de santé, a été conduite de manière plus intuitive selon une méthodologie plus proche de celles utilisées en sciences sociales, et sur base d'observations, d'évaluations et d'informations sanitaires recueillies en routine. Cette distinction est importante car elle autorise à argumenter en faveur du maintien d'activités de recherche, y compris selon une méthodologie complexe dans un environnement particulièrement peu stable.

Le contexte dans lequel ces recherches ont été menées a influencé grandement la manière dont les travaux ont été réalisés. En effet, cette partie du Congo est soumise à des troubles sévères et récurrents (guerre civile, crise humanitaire et éruptions volcaniques) depuis le débuts des années 1990. Le profil de l'état de santé des populations s'est considérablement aggravé, notamment en termes de mortalité, rendant les interventions essentielles mais également la recherche impérieuses afin d'améliorer l'efficience et la qualité de la prise en charge de ces populations. Ensuite, les problèmes d'organisation des services de santé sont également devenus beaucoup plus complexes et l'implication des organisations humanitaires d'urgence a dû être intégrée dans les paramètres d'intervention du Cemubac au Kivu. Il s'est agi clairement d'une justification essentielle en faveur du développement de la recherche en système de santé en situation de crise. Troisièmement, tout comme la formation, la recherche a été un des éléments moteurs dans le maintien à leur poste des cadres médicaux ou non médicaux, notamment parce que ces recherches amenaient des ressources additionnelles tant en moyens financiers, en 
matériel qu'en encadrement dans les hôpitaux et centres de santé. Les événements ont donc, de manière évidente, perturbé le déroulement de ces recherches en rendant souvent plus long le recueil des informations et des données mais a, par contre, apporté un soutien tout a fait opportun en termes de motivation professionnelle et financière et a permis de contribuer au maintien d'une cohésion parfois ténue dans la prestation des professionnels de santé locaux. La formation de nombreux cadres impliqués dans la prestation des soins et la gestion des services de santé a permis de garantir la continuité des interventions qui sont réalisées depuis 1990 sans présence expatriée permanente. Le soutien aux différents comités de santé permet aux professionnels de santé congolais d'assurer une intégration optimale au sein des communautés pour la compréhension des attitudes et pratiques de la population face aux problèmes de santé. Celle-ci est en effet représentée et active au sein de dizaines de comités de santé se réunissant chaque mois.

Nous envisagerons successivement les études de caractère quantitatif puis qualitatif. En ce qui concerne les études quantitatives, nous distinguerons trois grands types : les collectes de données de routine, les études épidémiologiques d'observation et les études épidémiologiques d'intervention.

\section{Les collectes de données de routine}

Par données de routine, on entend des informations sanitaires collectées au quotidien, synthétisées périodiquement et relatives au statut sanitaire (maladies, vaccinations, décès, etc.) des populations fréquentant les établissements sanitaires. Cette collecte se fait à travers des rapports mensuels mis au point avec l'aide des cadres locaux et analysés en concertation avec les représentants de la communauté. Les premières données collectées en routine datent de 1980. Il s'agissait de données collectées dans différentes maternités du Nord Kivu, qui incluaient des informations relatives à la femme, au nouveau-né et à la survie de la mère et de l'enfant. Dès 1980, ces données ont été récoltées à l'aide d'un format standardisé, ce qui a permis de centraliser et de compiler toutes les informations. Cette récolte de données est toujours effectuée actuellement et même si le format de recueil et les variables recueillies ont été modifiés au cours du temps, les données initiales sont restées compatibles avec les données plus récentes. L'ensemble des données constitue sans doute le recueil systématique de données le plus ancien mis en place dans une maternité africaine de milieu rural. Il a permis d'apporter des informations précieuses sur l'évolution de pratiques telles que la césarienne, sur l'évolution du poids de naissance et de la mortalité des enfants, sur l'état nutritionnel des mères ainsi que sur leur niveau d'études. Ces données ont permis de suivre à quel point la situation sanitaire restait critique pour les femmes et leurs enfants (Mugisho et al. 2002a, 2002b).

Quelques années plus tard, en 1986, une recherche sur la mortalité intra hospitalière menée à l'hôpital de Lwiro était le point de départ du recueil systématique de données socio-économiques, nutritionnelles et infectieuses d'admission des enfants ainsi que de données relatives au suivi des enfants et à leur état de sortie (Dramaix et al 1993, 1996).

Les analyses de ces données toujours recueillies actuellement ont permis notamment de voir combien la mortalité avait diminué au cours du temps grâce aux modifications qui avaient été apportées dans le traitement des enfants. Elles ont également permis d'établir des modèles qui, utilisés en routine, pourront désormais mettre en évidence rapidement les éventuels problèmes de prise en charge des enfants (Bahwere 2002).

Actuellement, des Systèmes d'information sanitaire (SIS) ont été mis en place par le Cemubac au Nord Kivu et à Kinshasa. Au Kivu, ces SIS permettent le recueil depuis 1985 - 
c'est-à-dire sur une période de plus de vingt ans - d'une masse considérable d'informations chiffrées relatives notamment aux performances des différents établissements sanitaires mais également aux ressources humaines et financières nécessaires pour assurer le fonctionnement des services de santé dans un environnement rendu particulièrement difficile par les conditions sécuritaires. L'exploitation des résultats qui en sont extraits est réalisée au quotidien par les équipes de cadres médicaux et non médicaux congolais au Nord Kivu et à Kinshasa (Porignon 2003).

\section{Les études épidémiologiques d'observation}

Les études scientifiques rigoureuses qui débouchent sur des actions bénéfiques pour la population font partie d'une tradition de longue date au Cemubac. Les études d'observation qui relèvent plutôt de la description des phénomènes ont été initiées très tôt. En effet, les premières études de ce type remontent à la fin des années 1970 au cours desquelles une recherche portant sur les mères allaitantes et leurs enfants a été menée au Sud Kivu. Cette recherche comportait une étude transversale (étude réalisée de façon ponctuelle à un moment donné dans une population) qui a mis en évidence l'état nutritionnel précaire des mères allaitantes et la situation particulièrement défavorisée des femmes du milieu rural. Cette même étude a également montré que cet état nutritionnel déplorable avait de lourdes conséquences sur la sécrétion lactée et par la même occasion sur l'état nutritionnel des enfants. Le second volet de cette recherche était constitué par une étude de cohorte (étude qui inclut un groupe de sujets suivis pendant une période donnée, pas forcément la même pour chacun) au cours de laquelle près de mille enfants allaités ont été suivis durant deux ans. Cette étude a permis de souligner la situation de malnutrition grave dans laquelle se trouvaient les enfants allaités parmi lesquels la mortalité était particulièrement élevée (Hennart 1983).

Le suivi des enfants hospitalisés à Lwiro entre 1986 et 1988 est une autre étude de cohorte : elle a consisté à suivre jusqu'à leur sortie plus de mille enfants admis à l'hôpital de Lwiro, pour la grande majorité d'entre eux (75\%) en malnutrition grave. Les résultats de cette étude ont montré un taux de mortalité intra-hospitalière élevé. Ces résultats ont également fait apparaître sur base d'un modèle de prédiction de la mortalité, la possibilité d'identifier dès l'admission les enfants les plus à risque de décès et d'intervenir de manière plus spécifique en leur administrant non seulement un traitement à base d'antibiotiques, mais également un régime de réhabilitation alimentaire adapté tenant compte des opportunités nutritionnelles locales (Bahwere 2002). Dans ce cadre, un accent particulier a été mis sur la vitamine A, qui a permis d'en démontrer la carence sub-clinique ainsi que les effets délétères de cette dernière (Donnen et al., 1996).

$\mathrm{Au}$ Nord Kivu, diverses études transversales ou longitudinales se sont portées sur la santé du couple «mère-enfant ». L'accessibilité à l'eau potable, le suivi de la croissance ainsi que le contenu de la consultation préscolaire, les conditions des accouchements ont été identifiés comme des facteurs importants jouant sur la fréquence des diarrhées, le statut nutritionnel des enfants de moins de cinq ans et la mortalité périnatale ou maternelle (Tonglet et al., 1992a, 1999; Mugisho 2002a et b).

\section{Les études épidémiologiques d'intervention}

Les études d'intervention au cours desquelles un ou plusieurs groupes reçoivent le ou les traitements testés et sont comparés à un groupe contrôle qui reçoit un placebo ou le traitement standard, sont celles qui permettent le plus « sûrement » de conclure à l'effet 
d'un traitement. Plusieurs études de ce type ont été menées dans le cadre des recherches $\mathrm{du}$ Cemubac. Pratiquement toutes ces études respectaient les règles d'un essai clinique théoriquement idéal : elles étaient randomisées (les sujets étaient alloués aléatoirement dans le groupe traitement ou le groupe placebo), en double aveugle (ni le patient, ni celui qui l'administrait ne savait s'il s'agissait du traitement ou du placebo) et contrôlées (il y avait toujours un groupe contrôle qui servait de comparaison). En outre, l'aspect éthique a toujours été pris en compte dans la mesure où tous les protocoles de ces études ont été approuvés par un comité d'éthique avant d'être mis en place.

Deux études sur l'impact de la supplémentation en vitamine A ont été réalisées au Sud Kivu, soit au sein de la communauté, soit en milieu hospitalier à Lwiro. Elles ont permis de mesurer l'efficacité de ce micronutriment dans le cadre de la prise en charge des maladies infectieuses, nombreuses dans la région (Donnen et al., 1998a et b). Au Nord Kivu, un essai clinique conduit chez des femmes enceintes avait comme objectif de comparer les effets du traitement préventif traditionnel (chloroquine) de la malaria à ceux du traitement au Fansidar, sur l'état de la femme, du nouveau-né et de sa croissance jusqu'à douze mois (Mugisho et al., soumis pour publication).

\section{Les aspects qualitatifs}

Bien qu'étant complémentaire aux études quantitatives, la démarche utilisée pour étudier le fonctionnement des systèmes de santé repose sur une dynamique néanmoins différente de celles décrites ci-dessus. Dans le domaine de l'organisation des systèmes et des services de santé, la démarche déductive est quasi utopique (Fulop et al. 2001). En effet, il est très peu probable, y compris pour des raisons éthiques, d'arriver à contrôler les différents facteurs qui peuvent jouer sur les variables étudiées rendant ainsi hasardeuses les conclusions tirées. L'absence de standardisation des conditions environnementales, l'implication plus importante du chercheur dans le processus d'investigation, la faible reproductibilité ainsi que la singularité dans la narration des expériences nécessaire pour assurer une meilleure compréhension de phénomènes complexes ancrés au sein de la communauté sont autant de facteurs qui freinent la généralisation des résultats obtenus. La démarche méthodologique de recherche en matière de système de santé, souvent plus inductive, s'appuie sur des constructions composées faisant appel à des méthodes multiples recouvrant en partie les aspects épidémiologiques illustrés ci-dessus et des aspects plus proches des sciences sociales. Alors que les chercheurs proches du paradigme positiviste/ déductif seront plus enclins à privilégier l'expérimentation telle que dépeinte ci-dessus, ceux choisissant l'approche interactionniste/inductive seront amenés à plus d'observation et de description de la signification ou du sens du monde social. Le standard pour les premiers sera l'étude expérimentale contrôlée au cours de laquelle le chercheur va suivre les effets du changement opéré. Les seconds prendront le contexte naturel ou quotidien comme source de données. Pour essayer de concilier ces deux approches ayant conduit à des oppositions très fortes entre chercheurs, ou peut-être plus simplement pour mieux appréhender la réalité, certains auteurs ont développé des approches alternatives comme le réalisme liant à la notion de causalité (au sens expérimental du terme) celle de contexte ou d'environnement (Pawson et Tilley 1997; Fulop et al. 2001). Les méthodes auxquelles nous avons eu recours durant la conduite des différents volets des recherches menées dans ce domaine par le Cemubac sont : l'observation participante, l'exploitation de documents et l'analyse de contenu, la recherche-évaluation, la méthode discrète et l'analyse de 
politique ou policy analysis (Rice et Ezzy 1999, Jones 2000, Fulop et al. 2001, Rosenhead et Mingers 2001). Ces méthodes ne sont évidemment pas mutuellement exclusives : au contraire, leur combinaison appropriée permet un renforcement mutuel des résultats.

Les résultats obtenus ont permis (1) de mettre en évidence les potentialités réelles d'un soutien aux services de santé locaux en situation de crise (Porignon et al. 1995, Porignon 2003), (2) de relever des problèmes liés à l'iniquité de l'aide internationale apportée, notamment durant la crise des réfugiés rwandais de 1994 à 1996 (Porignon et al. 2000) mais également (3) de s'intéresser aux aspects éthiques de l'implication des professionnels de santé dans la résolution des conflits, notamment en terme de documentation, de formation ou de contribution à la reconstruction du cadre sociétal à travers la mise en place des comités de gestion et de santé dans les différents établissements sanitaires (Porignon et al. 2004). L'analyse documentaire, la recherche historique et les interviews permettent d'affiner les conclusions tirées par ailleurs.

\section{Les outils utilisés}

Dans les études quantitatives qui ont été menées, les outils de collecte des données sont basés sur des protocoles établis de manière aussi rigoureuse que possible pour satisfaire les exigences de la communauté scientifique. Les protocoles sont établis par les chercheurs du nord ou du sud en fonction du sujet et des hypothèses formulées. Ils sont revus et discutés au cours des missions d'encadrement des responsables scientifiques du Cemubac sur le terrain ou au cours de séjours d'étude des chercheurs à Bruxelles. Ils sont donc basés sur un échange étroit entre les compétences techniques et scientifiques des collaborateurs du Cemubac à Bruxelles et sur la capacité technique, scientifique mais également opérationnelle des collègues œuvrant sur le terrain. Ces protocoles décrivent en détail (1) la nature, l'objectif et l'hypothèse de la recherche, (2) la procédure de recrutement des sujets souvent établie sur une base soit de routine, soit aléatoire, (3) le nombre de sujets nécessaires, (4) la nature de l'intervention qui sera réalisée (protocole thérapeutique ou de réhabilitation nutritionnelle par exemple), (5) les résultats attendus et l'utilité de la recherche.

L'enrôlement de patients dans une étude fait l'objet d'un consentement de la part de la personne. Si nécessaire, l'avis du Comité d'éthique de la Faculté de médecine de l'Université libre de Bruxelles est demandé.

Lors de la mise en œuvre de la recherche, des compétences additionnelles sont organisées à travers la formation afin d'assurer un recueil des informations correspondant aux standards scientifiques requis et garantissant ainsi la validité des études menées. Une des caractéristiques de ces recherches est le fait qu'elles sont le plus souvent initiées par la perception venant des professionnels de santé ou de la population d'un problème particulier. Les cadres de concertations mis en place en République Démocratique du Congo à travers les différents comités de santé permettent à la population d'exprimer ses attentes relatives à tel ou tel problème de santé. Ceux-ci, s'ils sont considérés comme pertinents, peuvent alors être relayés et traduits en protocole de recherche. L'exemple de la mortalité maternelle, ou celui des conditions d'organisation des services en période de crise, notamment en matière d'accessibilité aux soins, sont illustratifs à cet égard. Il convient donc de souligner que les attentes ou demandes émanant de la population proviennent plus d'une collecte quasi permanente à travers les contacts quotidiens et les réunions des différents comités de santé que de l'organisation épisodiques d'entretiens plus ou moins dirigés tels que pratiqués dans la recherche en sciences humaines. 
La collecte d'information de routine s'opère au sein des structures sanitaires grâce au personnel de l'établissement qui a été spécialement formé et dont c'est l'une des responsabilités quotidiennes. L'enregistrement systématique d'informations concernant des problèmes spécifiques se fait sur base de registres. L'enregistrement des accouchements dans les maternités en est un exemple. L'ensemble des activités communes (consultations curatives, vaccinations, consultations prénatales, consultations préscolaires, planification familiale, accouchements, prise en charge des malades chroniques, etc.) est répertorié au sein de chaque établissement sanitaire et fait l'objet d'un rapport mensuel transmis à la hiérarchie du district sanitaire (zone de santé). La compilation et l'analyse de ces données constituent une source importante pour l'étude des performances du système de santé dans ses différents aspects. Ces études sont menées par les cadres congolais avec le soutien de l'équipe du Cemubac à Bruxelles. A ce niveau, l'implication des professionnels congolais est très importante puisque ce sont eux qui gèrent et utilisent ces données au quotidien dans un processus de réflexion critique soutenu par le Cemubac.

La méthodologie ainsi développée par le Cemubac à travers les interventions sanitaires et la recherche au Kivu, alliant des aspects quantitatifs (suivi longitudinal, analyse de tendances, comparaisons, analyse multivariée, analyse économique, etc.) et qualitatifs (observation participante, analyse documentaire, policy analysis, méthode discrète, etc.) vise à argumenter (1) la complémentarité entre les deux types d'approche ainsi que (2) la richesse potentielle de la recherche médicale appliquée à des problèmes de santé spécifiques (malnutrition, malaria, etc.) ou à l'organisation des services de santé, y compris dans des environnements complexes (Grodos et Mercenier 2000, Jones 2000, Creswell 2003, Ritchie et Lewis 2003).

\section{Eléments de discussion}

Comme l'entend la stratégie de coopération du Cemubac, l'implication de partenaires locaux est au cœur du processus d'intervention mais aussi de recherche. Cette implication repose sur un ensemble de caractéristiques: (1) le recrutement de chercheurs réalisé de manière non sélective, c'est-à-dire au hasard des nominations effectuées par les autorités sanitaires et administratives affichant ainsi la volonté de respecter les choix opérés par ces autorités en matière de gestion des ressources humaines; (2) le maintien d'une ligne de conduite visant à répondre aux questions de recherche relevant de problématiques sanitaires locales identifiées par les collaborateurs/chercheurs euxmêmes; (3) l'implication immédiate de ces collaborateurs médicaux et non médicaux dans une démarche de réflexion critique basée sur les activités quotidiennes expliquant l'importance accordée aux données de routines; (4) l'enrichissement de ces acquis à travers des formations formelles réalisées localement, en République démocratique du Congo, dans la sous-région ou en Europe; (5) l'encadrement rapproché par des équipes du Cemubac Bruxelles ${ }^{1}$ qui permettent de mettre en œuvre des recherches « incarnées » s'appuyant sur les structures existantes et valorisant le potentiel humain considérable qui preste encore dans beaucoup d'endroits dont les provinces du Kivu.

L'ensemble des éléments relevés ci-dessus a permis de développer une capacité et des compétences suffisantes pour que les acteurs congolais gèrent, depuis 1990, la

1. Cet encadrement se fait dans l'ensemble des domaines concernés qu'ils soient cliniques, organisationnels, gestionnaires ou relevant de la recherche. 
plupart des activités sur le terrain sans présence expatriée permanente. Il a permis, par sa progressivité, de mettre en place une dynamique d'apprentissage, de collection de savoirs et d'acquisition de connaissances dans des domaines divers allant de la médecine clinique aux sciences politiques en passant par les politiques de santé ou la gestion de projets. Il s'agit donc du cadre instruisant des conditions qui ont autorisé non seulement le maintien par le Cemubac, malgré les énormes difficultés qu'a connues la région du Kivu, non seulement des activités au bénéfice des populations mais encore l'entretien d'une réelle dynamique de réflexion critique et de recherche. Le cadre à la fois corrélatif et suscitant servi par un accompagnement technique et logistique permet de créer un partenariat aboutissant au raffermissement des liens de confiance et de respect mutuel, qui contribue à motiver et à retenir sur place les cadres formés. La lutte contre le brain drain est actuellement reconnue, par l'ensemble de la communauté internationale, comme un élément majeur pour la reprise d'une dynamique globale de développement (World Bank 2003). Par ailleurs, la combinaison dans la formation des ressources humaines d'un volet opérationnel et d'un volet recherche appliquée apporte une valeur ajoutée quant à l'efficacité, à l'appropriation et à la crédibilité des interventions menées sur le terrain. La plupart des projets et des questions de recherche sont formulés par les acteurs de terrain, réalisés par eux en concertation de plus en plus large avec les autorités sanitaires et avec les partenaires en place. Les données et informations collectées, notamment en routine, servent de bases pour la prise de décision et la mise en œuvre des activités tant au Nord qu'au Sud Kivu. Il s'agit donc de l'output réel d'un processus alliant intervention et recherche localement appropriée.

Si la participation et l'implication des cadres locaux sont réelles, le soutien aux structures de base permet également de tenir compte des attentes de la population, comme ce fut le cas notamment pour la problématique de l'approvisionnement en eau potable (Tonglet et al. 1992a), de la lutte contre la malnutrition (Tonglet et al. 1992b), l'augmentation de la couverture en infrastructures (Porignon et al. 1998) ou les recensements de population (Reynders et al. 1992).

\section{Conclusions}

La recherche médicale appliquée ainsi que la recherche en organisation des services et des systèmes de santé sont réalisables, même (peut-être surtout) en situation critique. Elle peuvent se démarquer d'interventions de développement au sein desquelles elles sont ancrées par la rigueur méthodologique utilisée pour mener des études quantitatives et qualitatives, par la formulation d'hypothèses, par l'utilisation de méthodes diverses et complémentaires et par la triangulation des arguments apportés.

La recherche développée par le Cemubac au Kivu a permis d'acquérir des connaissances dans des domaines divers (aussi bien cliniques qu'organisationnels), de contribuer à la motivation des cadres sanitaires favorisant leur maintien dans un environnement particulièrement complexe et de créer ainsi une dynamique dont le but principal reste de fournir des soins aux populations qui, dans cette partie du monde particulièrement, vivent en situation profondément critique depuis plus de vingt ans. 


\section{Références bibliographiques}

BAWHERE, J.-L., 2002. Contribution à l'amélioration et à l'évaluation de la prise en charge de l'enfant hospitalisé en Afrique centrale (République Démocratique du Congo). Thèse présentée en vue de l'obtention du grade de docteur en santé publique. Université libre de Bruxelles : Ecole de santé publique.

Creswell, J.W., 2003. Research Design: Qualitative, Quantitative, and Mixed Methods Approaches. California : Sage Publications, Thousand Oaks.

Donnen, P., D. Brasseur, M. Dramaix, F. Vertongen, Zihundila Mweze, Ngoy Bauma et P. Hennart, 1996. «Vitamin A deficiency and protein-energy malnutrition in a sample of pre-school age children in the Kivu Province in Zaire », European Journal of Clinical Nutrition, 50, pp. 456-61.

Donnen, P., D. Brasseur, M. Dramaix, F. Vertongen, Zihundila Mweze, M. Mbasha et P. Hennart, 1998a. «Vitamin A supplementation but not deworming improves growth of preschool malnourished children in eastern Zaire », Journal of Nutrition, 128, pp. 1320-27.

Donnen, P., D. Brasseur, M. Dramaix, R. Bitwe, F. Vertongen et P. Hennart, 1998b. « Randomized placebocontrolled clinical trial of the effect of a single high-dose or daily low-doses of vitamin A supplementation on morbidity of hospitalized malnourished children », American Journal of Clinical Nutrition, 68, pp. 1254-60.

Dramaix, M., P. Hennart, B. Paluku, O. Mudjene, R. Smets, P. Donnen, R. Tonglet et D. Brasseur, 1993. «Valeur des indicateurs nutritionnels pour le pronostic de la mortalité intra-hospitalière chez les enfants du Kivu », Revue d'épidémiologie et de santé publique, 41, pp. 131-8.

Dramaix, M., D. Brasseur, P. Donnen, P. Bawhere, D. Porignon, R. Tonglet et P. Hennart, 1996. « Prognostic indices for mortality of hopitalized children in Central Africa », American Journal of Epidemiology, 143 (12), pp. 1235-43.

Fulop, N., P. Allen, A. Clarke et N. Black, 2001. « Issues in studying the organisation and delivery of health services », in N. Fulop, P. Allen, A. Clarke and N. Black (eds), Studying the Organisation and Delivery of Health Services. Research Methods. Londres : Routledge.

Grodos, D. et P. Mercenier, 2000. « La recherche sur les systèmes de santé : mieux comprendre la méthodologie pour mieux agir », Studies in Health Services Organisation \& Policy, 14, pp. 1-200. Anvers : Institut de médecine tropicale.

Hennart, P., 1983. Allaitement maternel en situation nutritionnelle critique : adaptations et limites. Thèse présentée en vue de l'obtention du grade d'agrégé de l'enseignement supérieur. Lwiro - Université Libre de Bruxelles : Cemubac et IRS.

Jones, R. A., 2000. Méthodes de recherche en sciences humaines. Bruxelles : De Boeck.

Mugisho, E., M. Dramaix, D. Porignon, E. Musubao et P. Hennart

2002a. «Evolution des données maternelles et périnatales recueillies en routine entre 1980 et 1998 à la maternité de référence de Rutshuru en République Démocratique du Congo I. Décès maternels et interventions obstétricales », Cahiers Santé, 12, pp. 247-251.

2002b. «Evolution des données maternelles et périnatales recueillies en routine entre 1980 et 1998 à la maternité de référence de Rutshuru en République Démocratique du Congo II. Décès du nouveau-né et naissances de faible poids », Cahiers Santé, 12, pp. 252-255.

Mugisho, E, S. Meuris, P. Hennart, D. Porignon et M. Dramaix, (soumis pour publication). « Effects of antenatal malaria prophylaxis (sulfadoxine-pyrimethamine vs chloroquine) on the growth of the newborn during the first year in North Kivu, Democratic Republic of Congo : a randomized clinical trial ». Soumis pour publication dans Tropical Medicine \& International Health.

Pawson, R., et N. Tilley, 1997. Realistic Evaluation. Londres : Sage Publications.

Porignon, D., 2003. Adéquation des systèmes de santé de district en situation critique. Expériences dans la Région des Grands Lacs africains. Thèse présentée en vue de l'obtention du grade de docteur en sciences de la santé publique, Ecole de santé publique, Université libre de Bruxelles. 
Porignon, D., E. Mugisho Soron'Gane, T. Elongo Lokombe, D. Katulanya Isu, P. Hennart et W. Van LERBERGHe, 1998. « How robust are health district systems? Coping with crisis and disasters in Rutshuru, Democratic Republic of Congo », Tropical Medicine \& International Health, 3 (7), pp. 559-65.

Porignon, D., D. Katulanya Isu, J. Ntalemwa Nytha, R. Tonglet, M. Dramaix et P. Hennart, 2000. « The unseen face of humanitarian crisis in Eastern Democratic Republic of Congo : was nutritional relief properly targeted? », Journal of Epidemiology and Community Health, 54 (1), pp. 6-9.

Porignon, D., E. Mugisho et P. Hennart, 2004. « La santé publique, un volet essentiel pour l'élaboration d'un processus de paix. Stratégies et acteurs d'une discipline émergente », in Pierre Calame, Benjamin Denis et Eric Remacle (éds), L'art de la paix : approche transdisciplinaire, pp. 115-132. Berne, Bruxelles : PEI Peter Lang.

Reynders, D, R. Tonglet, E. Mahangaiko Lembo, D. Mertens, Katulanya Isu, M. Dramaix et P. Hennart, 1992. «Les agents de santé communautaires sont capables de déterminer avec précision la populationcible des programmes de santé », Annales de la Société belge de médecine tropicale, 75, pp. 145-54.

Rice, P. et D. Ezzy, 1999. Qualitative Research Methods. A Health Focus. Oxford : Oxford University Press.

Ritchie, J. et J. Lewis, 2003. Qualitative Research Practice. Londres : Sage Publications.

Rosenhead, J. \& J. Mingers, 2001. Rational Analysis for a Problematic World Revisited. Problem Structuring Methods for Complexity, Uncertainty and Conflict. Chichester : John Wiley and sons Ltd.

Tonglet, R., I. Katulanya, M. Munkatu, M. Dramaix et P. Hennart, 1992a. « Can improvements in water supply reduce childhood diarrhoea? », Health Policy and Planning, 7, pp. 260-8.

Tonglet, R., M. Maheshe, B. Masumbuko, I. Beghin et P. Hennart, 1992b. « The causal model approach to nutritional problems : an effective tool for research and action at the local level », Bulletin of the World Health Organization, 70 (6), pp. 715-23.

Tonglet, R., L. E. Mahangaïko, Z. Mweze, A. Wodon, M. Dramaix et P. Hennart, 1999. « How useful are anthropometric, clinical and dietary measurements of nutritional status as predictors of mortality of young children in central Africa? », Tropical Medicine and International Health, 4 (2), pp. 20-130.

World BAnk, 2003. World Develoment Report 2003. World Bank, Oxford : Oxford University Press. 
